\title{
The relationships between resilience and student personal factors in an undergraduate medical program
}

\author{
Ardi Findyartini ${ }^{1,2^{*}}$, Nadia Greviana ${ }^{1,2}$, Azis Muhammad Putera ${ }^{2,3}$, Reynardi Larope Sutanto ${ }^{2,3}$, \\ Vernonia Yora Saki ${ }^{1}$ and Estivana Felaza ${ }^{1,2}$
}

\begin{abstract}
Background: Resilience is an essential aspect of wellbeing that plays a major role in undergraduate medical education. Various personal and social factors are known to affect resilience. Empirical evidence remains limited regarding resilience and the personal factors that affect it among undergraduate medical students in an Asian setting. Therefore, this study aims to identify undergraduate medical students' level of resilience and its relationships to personal factors in Indonesia.
\end{abstract}

Methods: This cross-sectional study was conducted among undergraduate medical students in years 1-6. Respondents were asked to complete three validated questionnaires: the Connor-Davidson Resilience Scale (CDRISC) to measure resilience, the Brief-COPE to assess coping mechanisms, and the Big Five Personality Test to measure five personality dimensions. Descriptive and Pearson's correlation analyses were completed to explore relationships between each variable. Regression analysis was completed to analyze the extent to which coping mechanisms, personality, and academic achievement explained the variation in resilience scores.

Results: A total of 1040 respondents completed the questionnaires (a $75.42 \%$ response rate). Students in both preclinical and clinical stages had quite good levels of resilience and higher scores on adaptive coping mechanisms than on maladaptive coping mechanisms. Adaptive and maladaptive coping mechanisms, Big Five Personality traits (extraversion, agreeableness, conscientiousness, neuroticism, and openness), and students' academic achievement explained $46.9 \%$ of students' resilience scores.

Conclusions: Although the resilience scores in this study were comparable to resilience scores among undergraduate medical students in other settings, we found that coping mechanisms, personality traits, and academic performance may predict resilience among medical students.

Keywords: Resilience, Undergraduate, Medical students, Coping mechanism, Personality traits

\footnotetext{
* Correspondence: ardi.findyartini@ui.ac.id; findyartini@yahoo.com

${ }^{1}$ Medical Education Center, Indonesia Medical Education and Research

Institute (IMERI), Faculty of Medicine Universitas Indonesia, Jakarta, Indonesia

${ }^{2}$ Department of Medical Education, Faculty of Medicine Universitas

Indonesia, Jakarta, Indonesia

Full list of author information is available at the end of the article
}

(c) The Author(s). 2021 Open Access This article is licensed under a Creative Commons Attribution 4.0 International License, which permits use, sharing, adaptation, distribution and reproduction in any medium or format, as long as you give appropriate credit to the original author(s) and the source, provide a link to the Creative Commons licence, and indicate if changes were made. The images or other third party material in this article are included in the article's Creative Commons licence, unless indicated otherwise in a credit line to the material. If material is not included in the article's Creative Commons licence and your intended use is not permitted by statutory regulation or exceeds the permitted use, you will need to obtain permission directly from the copyright holder. To view a copy of this licence, visit http://creativecommons.org/licenses/by/4.0/ The Creative Commons Public Domain Dedication waiver (http://creativecommons.org/publicdomain/zero/1.0/) applies to the data made available in this article, unless otherwise stated in a credit line to the data. 


\section{Background}

Resilience has been recognized as an essential aspect of wellbeing in medical education that enables students to recover from adversities and challenges $[1,2]$. The concept of resilience can be seen from psychological, sociological, ethical, and moral perspectives. Thus, resilience has been defined as "a dynamic capability which allows people to thrive on challenges given appropriate social and personal contexts" [2]. Despite the dynamic nature of resilience and its multidimensional factors [3, 4], it is believed that resilience can be learned [3]. More importantly, resilience can also be promoted among individuals, resulting in better psychological and physical states [4].

Resilience plays a major role among undergraduate medical students due to their heavy workloads, which are associated with curricula, transitions in medical training, and changes in their personal lives. Compared to other student groups of the same age, medical students are more likely to endure negative psychological states, such as depression, burnout, psychosomatic complaints, decreased empathy, thoughts about quitting school, self-harm, and suicidal ideation, as well as poor academic performance $[5,6]$. One study found that $27.2 \%$ of medical students were depressed or showed depressive symptoms, while $11.1 \%$ of medical students showed suicidal ideation [7]. Therefore, an emphasis on cultivating resilience is critical for medical students to mitigate and cope with the negative effects of their stressors [3, 8].

Various factors may affect the development of resilience among medical students. Social support and coping styles are two of these factors [3]. A cross-sectional study conducted in Brazil found that older students, those with a good perception of health, and students refusing to use habit-forming medications all demonstrated increased resilience [5]. Personality type is also suggested as a factor in resilience. Based on the Big Five Personality framework, personality traits that support better resilience are openness, agreeableness, conscientiousness, and extraversion due to better social support, adaptive coping, and positive emotions. On the other hand, neuroticism, which is related to negative emotions and maladaptive coping, tends to reduce resilience [9, 10]. Academic performance reportedly correlates with students' resilience via academic satisfaction [11]. Another study also suggested that students' past performance can predict future course performance for highand low-achieving students. While self-assuredness is an individual factor that may improve performance among low achievers, drive is a predictor of performance among high achievers [12].

Current medical students from Generation $\mathrm{Z}$ are known to be more prone to insecurities, depression, and anxiety. Moreover, their overreliance on adults and information technology during their formative years may also affect their ability to cope with challenges in life [13]. It should be realized, however, that different societies have different values and norms that must be considered to understand the factors that influence resilience in the current generation of medical students [14]. Studies on medical students' resilience in Asian settings are limited [14-16]. Few studies have examined personal factors and their relationships to resilience among undergraduate medical students in settings where cultures and conditions are different from the West [17, 18]. Therefore, this study aims to identify the relationships between resilience and personal factors (personality, coping mechanisms, and academic performance) among undergraduate medical students in Indonesia. The research questions are as follows: a. What is the resilience level of undergraduate medical students from different years of study? b. How do medical students' personality, coping mechanisms, and academic performance affect their resilience? This study helps inform medical education in this setting to better support resilience throughout their medical training by considering individual factors.

\section{Methods \\ Context}

The undergraduate program of the Faculty of Medicine Universitas Indonesia (FMUI) uses a 5.5 year-competency-based curriculum. The medical school accepts high school graduates, who are required to take preclinical courses during the first 3.5 years (7 semesters). In the preclinical stage, the curriculum is developed progressively with a system-based learning approach. The primary learning activities during this stage include problem-based learning, collaborative learning, interactive lectures, and laboratory and clinical skills sessions, with sufficient time for independent learning.

The clinical stage begins in the eighth semester with a clinical rotations approach. Students are assigned to lengthy clinical rotations in internal medicine, surgery, obstetrics/gynecology, and pediatrics during their first year in the clinical stage, followed by shorter clinical rotations during the second year of their clinical stage. Following the short clinical rotations, students are placed in primary care centers and district hospitals for a preinternship program. The primary learning activities during the clinical stage are conducted in the workplace, where students are exposed to patient encounters under supervision, case presentations, and clinical skills sessions.

\section{Study design}

We utilized a cross-sectional design with a total sampling approach. Students in years 1-6 in preclinical and 
clinical stages were invited to complete questionnaires from December 2019 to January 2020. This study was approved by the Research Ethical Committee of FMUI and Dr. Cipto Mangunkusumo (National Hospital No. KET-1299/UN2.F1/ETIK/PPM.00.02/2019).

\section{Population and respondents}

Respondents in this study were undergraduate medical students at FMUI in preclinical and clinical stages with active enrolment status during academic year 2019/2020. The total number of FMUI undergraduate medical students from years 1-6 was 1379 students.

\section{Instrument}

Three questionnaires were administered in this study: the Connor-Davidson Resilience Scale (CD-RISC) measured students' resilience levels, the Brief COPE assessed coping strategies, and the Big Five Personality Inventory examined students' personality traits.

The Connor-Davidson Resilience Scale (CD-RISC) was developed by Kathryn M. Connor and Jonathan R.T. Davidson to measure resilience among Post-Traumatic Stress Disorder (PTSD) patients. The CD-RISC inventory that we selected is the 25-point inventory with a 0 4 scale. A higher score reflects a higher level of resilience [19]. Brief COPE is a 14-subscale inventory with two items for each subscale, developed by Charles S. Carver for assessing coping strategies. It is a shortened version of the previously developed Cope Inventory. The Brief COPE inventory was used to elucidate the coping mechanisms generally used by respondents. Brief COPE has a Cronbach's alpha of 0.68-0.90 [20]. The Big Five Personality is an inventory developed through a lexical approach; it attempts to categorize five dimensions of personality: extraversion, agreeableness, conscientiousness, neuroticism, and openness. To measure the level of each personality dimension for this study, respondents were asked to complete a 50-item questionnaire with 15 Likert scales [21].

Prior to their use in this study, both the CD-RISC and the Brief COPE inventories were translated from English to Bahasa Indonesia and back-translated to ensure consistency. Following back-translation, we compared the final Bahasa Indonesia versions of the questionnaires to the original versions to ensure their comparable meaning. The Big Five Personality questionnaire used in this study had previously been validated in an Indonesian setting [22].

In addition to questionnaires, we collected grade point average (GPA) data on respondents' academic achievements from the study program administrators; the maximum GPA is 4.00 .

\section{Data collection}

The questionnaires were distributed online through Google Forms for the preclinical students, while clinical students received hard copies from department administrators during their clinical rotations. All respondents provided informed consent to participate in this study.

\section{Data analysis}

The data were analyzed using SPSS version 21 . We assessed the reliability of all instruments. An alpha value higher than 0.9 indicates excellent internal consistency; a value of at least 0.7 indicates acceptable internal consistency [23]. The descriptive summary statistics (mean, proportion) were conducted. Descriptive analysis for CD-RISC was conducted by adding together all scores, with a higher score indicating a higher level of resilience (scores range from 0 to 100). The Brief COPE analysis was conducted by summing the subscales and regrouping them into categories of adaptive coping mechanisms: problem-focused (score of 6-24), emotionfocused (score of 10-40), and maladaptive coping mechanisms (score of 12-48) [24, 25]. We analyzed the Big Five Personality test by adding the scores separately for each personality dimension: extraversion (score 8-40), agreeableness (score 9-45), conscientiousness (score 945), neuroticism (score 8-40), and openness (score 1050) $[19,22]$. We performed an ANNOVA test to determine differences in the resilience, coping mechanisms, and personality scores of students in each year. Following the descriptive analysis, we conducted post-hoc analysis using the Tukey technique or the Honest Significant Difference (HSD) test to further analyze differences between student groups.

Using Pearson's analysis, we performed correlation analyses between resilience and each variable (coping mechanisms, the Big Five Personality test, and academic performance). We then analyzed the extent to which independent variables (coping mechanisms, personality, and academic achievement) may explain the variation in resilience scores.

\section{Results}

A total of 1040 respondents completed the questionnaires. The respondents' characteristics are shown in Table 1.

The internal consistency (as measured by Cronbach's alpha) of the CD-RISC and Brief COPE questionnaires used in this study are very good (Cronbach's alpha scores of 0.886 and 0.760 , respectively), whereas the Big Five Personality test has a Cronbach's alpha of 0.681 . Furthermore, according to the Kolmogorov-Smirnov normality test that we conducted for all subscales in all three questionnaires, the data from the results were 
Table 1 Respondents Characteristics

\begin{tabular}{llllll}
\hline Year & Male & Female & Total Respondents & Total Students & Response Rate (\%) \\
\hline 6 & $53(40.8)$ & $77(59.2)$ & 130 & 222 & 58.56 \\
5 & $64(40.3)$ & $95(59.7)$ & 159 & 222 & 71.62 \\
4 & $53(43.1)$ & $70(56.9)$ & 123 & 221 & 55.66 \\
3 & $93(48.4)$ & $99(51.6)$ & 192 & 232 & 82.76 \\
2 & $51(28.5)$ & $128(71.5)$ & 179 & 234 & 76.50 \\
1 & $85(36.0)$ & $151(64.0)$ & 236 & 248 & 95.16 \\
Grand Total & $\mathbf{3 5 9}$ & $\mathbf{6 6 0}$ & $\mathbf{1 0 4 0}$ & $\mathbf{1 3 7 9}$ & $\mathbf{7 5 . 4 2}$ \\
Percentage & 34.52 & 63.46 & 100 & - & - \\
\hline
\end{tabular}

normally distributed; therefore, all data in the results are described as means with standard deviations.

FMUI students in both preclinical and clinical stages had moderately high levels of resilience (Table 2), ranging from $64.76 \pm 10.69$ to $67.60 \pm 11.60$. Table 2 also shows the coping mechanisms used by students, as well as their GPAs and their personality types according to the Big Five Personality Test. The results suggest that the students had higher scores on adaptive coping (emotion-focused) than on maladaptive coping. Year-1 students had the highest scores; year-5 students had the lowest scores on extraversion, agreeableness, conscientiousness, and openness to experience. This also aligns with GPA scores: year-1 students had higher GPAs than other groups.

The correlation between personality and resilience was weak-to-moderate for extraversion, agreeableness, conscientiousness, and openness; there was no correlation between neuroticism and resilience. The correlation between coping mechanisms and resilience mostly showed moderate correlation. Students' GPAs across groups show a very low, yet statistically significant, correlation with resilience. (Table 3).

To predict students' resilience, we completed a regression analysis. The summary of the results is reported in Table 4. Overall, adaptive and maladaptive coping mechanisms, Big Five Personality traits (extraversion, agreeableness, conscientiousness, neuroticism, and openness), and GPA explained $46.9 \%$ of students' resilience.

The regression equation in this study is as follows:



The value of R Square (0.469) indicates that the effect of the independent variables (adaptive coping score, maladaptive coping score, GPA, extraversion, agreeableness, conscientiousness, neuroticism, and openness) on resilience (CD RISC) is $46.9 \%$. The remaining $53.1 \%$ is determined by other variables not considered in study.

According to Table 5, the model indicates that Brief COPE (Adaptive and Maladaptive), GPA, Big Five Personality traits (extraversion, agreeableness, conscientiousness, neuroticism, and openness) together explained $43.3-56.2 \%$ of resilience in year $1-6$ students.

\section{Discussion}

This study aims to identify the level of resilience among undergraduate medical students and its relationships with individual factors: personality, coping mechanisms, and academic performance. We found that the CD-RISC mean score ranged from 64.76 to 67.60 . The scores were higher than those from studies among medical students conducted in China (mean score 61.7) or Iran (mean score 62.11) $[17,18]$. Studies using CD-RISC in an Asian setting among university students also found a mean score of $60-70$ [26-28]. In other settings, such as the USA, UK, and Australia, the range is 70-90 [29-31]. According to Connor and Davidson [19], a higher CDRISC score indicates greater resilience. Our results fall within quartile 1 of the CD-RISC score (0-73), similar to other studies among medical students in an Asian setting. Culture and sociocultural context are believed to influence coping mechanism and resilience [32, 33] through dynamic interactions of personal traits, cultural values, cultural background and facilitating factors from the environment [32]. Therefore, current results of CDRISC in Asian setting with hierarchical, collectivist, masculine and uncertainty avoidance cultural context [34] might reflect the dynamic interactions of both personal and environmental factors which influence students' resilience. For example, expressing individual mental vulnerability might be discouraged in this culture due to competitiveness [35], and high intolerance in dealing with uncertainties coming from the learning environment [36] may progress to individual psychological problems which may reduce the resilience. In addition, the resilience as measured by CD-RISC comes from Western conceptual framework, hence the overall 


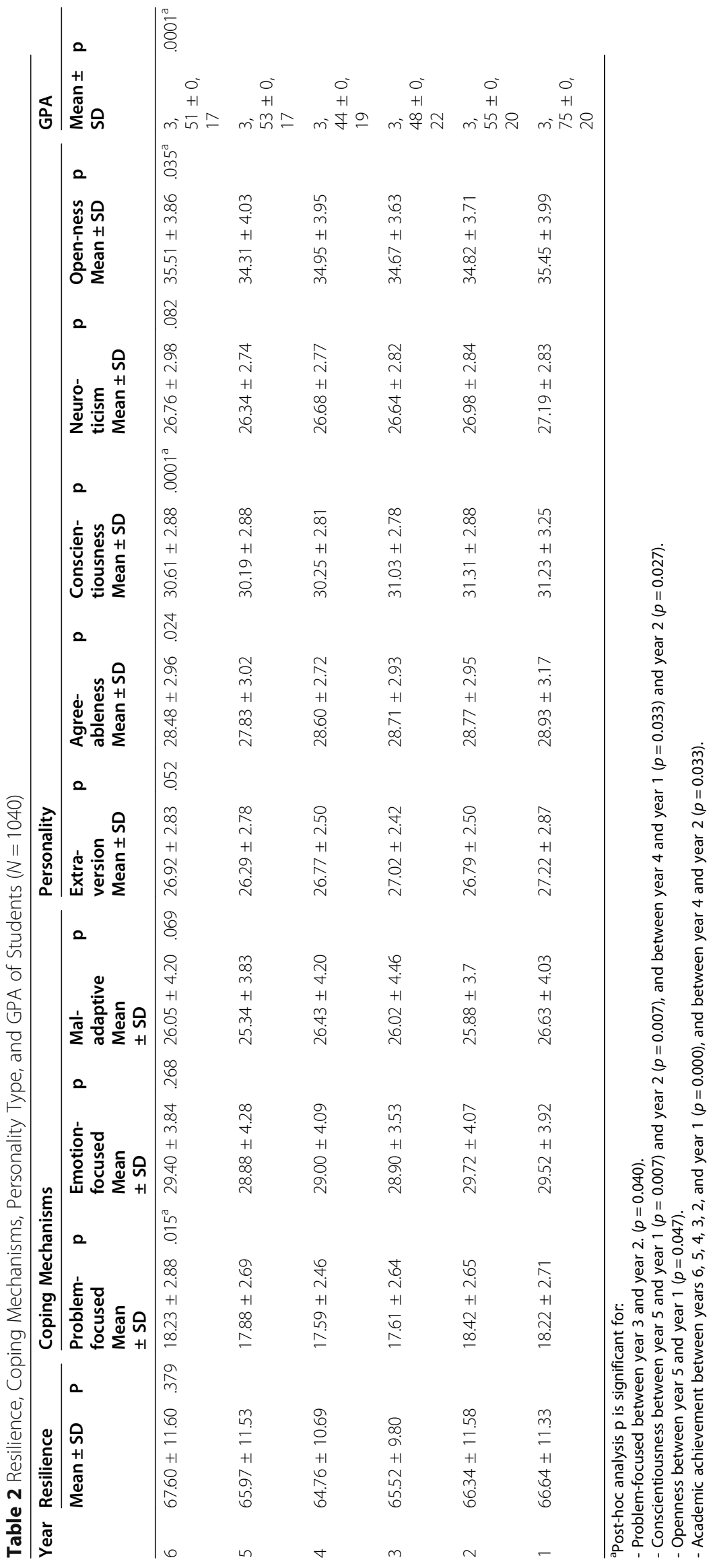




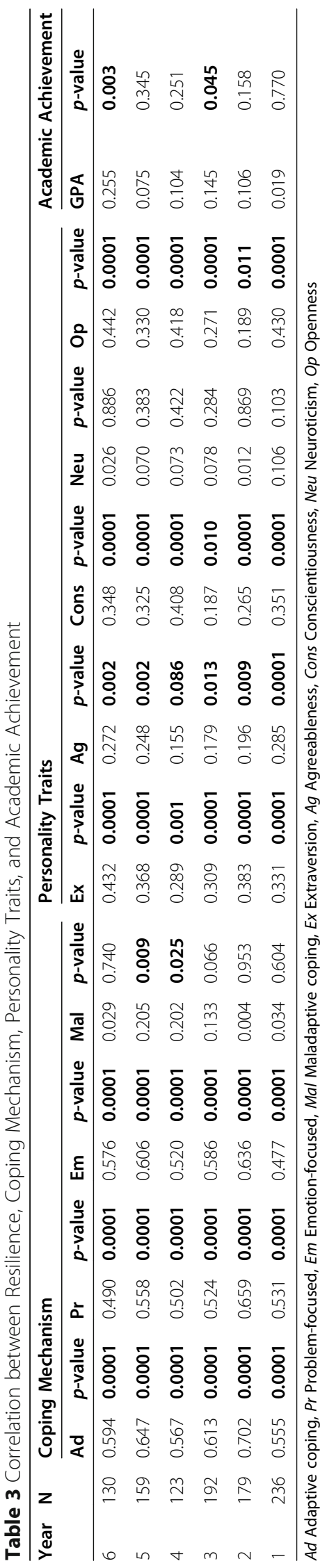


Table 4 Regression coefficients among variables

\begin{tabular}{llllll}
\hline Variables & Coefficient & $\mathbf{t}$ & $\boldsymbol{p}$-value & $\mathbf{R}^{\mathbf{2}}$ & $\mathbf{F}$ \\
\hline Constant & -4.125 & & & $\mathbf{. 4 6 9}$ & 113.905 \\
Adaptive Score & 0.960 & 21.065 & 0.0001 & & \\
Maladaptive Score & -0.459 & -7.119 & 0.0001 & & \\
GPA & 0.348 & .318 & 0.751 & & \\
Extraversion & 0.467 & 4.264 & 0.0001 & & \\
Agreeableness & 0.340 & 3.408 & 0.001 & & \\
Conscientiousness & 0.522 & 5.174 & 0.0001 & & \\
Neuroticism & -0.476 & -4.846 & 0.0001 & & \\
Openness & 0.288 & 3.808 & 0.0001 & & \\
\hline
\end{tabular}

construct might not be transferable in non-Western setting. This calls for further study on understanding resilience concept from multicultural perspectives, especially in non-Western setting [37].

We found that the mean resilience score of year-1 students (66.64) was higher than that of students in years $2-4(66.34,65.52$, and 64.76 , respectively). The mean resilience scores of students in years 5 and 6 seemed to rebound (65.97 and 67.60, respectively). Nevertheless, the mean score difference was not statistically significant; due to the cross-sectional design, we could not report the score change for individual cohorts. Regarding the curriculum, year-4 students were transitioning between preclinical and clinical years, which necessitates adaptation during this period [38] and may entail difficult clinical events [8]. Studies on empathy among undergraduate medical students suggest a similar pattern due to the challenges faced during this transition time $[39,40]$. However, the 'bounce-back' phenomenon for empathy has been reported in studies in Asia, suggesting the importance of a supportive system and adaptation in a clinical environment [41, 42].

Medical students in years 1-6 showed relatively similar patterns of Big Five Personality Traits. Compared to students from other year levels, Year-1 students showed a greater propensity for fulfilment from external sources (extraversion), more attempts to adjust their behavior to suit others (agreeableness), a high tendency to follow rules (conscientiousness), and a greater willingness to

Table 5 Regression analysis of factors contributing to resilience, by year

\begin{tabular}{llll}
\hline Year & $\mathbf{B}$ & $\mathbf{F}$ & $\mathbf{R}^{\mathbf{2}}$ \\
\hline 6 & -27.510 & 13.732 & 0,476 \\
5 & -12.035 & 21.565 & 0.535 \\
4 & 20.321 & 18.280 & 0.562 \\
3 & -5.747 & 22.624 & 0.497 \\
2 & -12.754 & 24.256 & 0.533 \\
1 & 9.507 & 21.712 & 0.433 \\
\hline
\end{tabular}

seek new experiences (openness). There were also consistent positive weak-to-moderate correlations between some personality types (extraversion, agreeableness, conscientiousness, and openness) and resilience in medical students across years 1-6. On the other hand, neuroticism did not correlate significantly with resilience. This result aligns with a meta-analysis on the relationship between Big Five personality traits and resilience [43]. The Big Five Personality Test incorporates personality and social cognitive theories [44], which helps explain why self-efficacy is essential for explaining the positive correlation between resilience and the personality traits extraversion, agreeableness, conscientiousness, and openness. Extraversion fosters positive reactions from others [45]; agreeableness helps people initiate new activities and achieve expertise [46]; conscientiousness increases task engagement and effort [47]; openness helps redefine demands as challenges to be overcome [48], which heightens self-efficacy in overcoming adversity. Conversely, neuroticism increases anxiety, which reduces self-efficacy [49].

Coping mechanism, defined as individual's cognitive and behavioural attempts to deal with demand that are perceived as stressful [50], mostly showed moderate correlations with resilience in our study. Similar results were shown in studies conducted among medical students in South Africa [51], China [16] and United States [3]. Based on the well-being model [52], students' internal factors, such as personality traits, temperament, and coping styles - acts as a reservoir in dealing with stressors. Students' choice of coping styles would later affect the resulting condition of being in a state of burnout or becoming a resilient character. The optimal conditions to develop resilience take place when stressors are adequate to be considered as challenges [53]. Moreover, students from all years were found to have higher scores for adaptive coping (emotion-focused scores of $28.88 \pm 4.28$ to $29.72 \pm 4.07$; problem-focused scores of $17.59 \pm 2.46$ to $18.42 \pm 2.65$ ) than for maladaptive coping (scores of $25.34 \pm 3.83$ to $26.63 \pm 4.03$ ). Interestingly, students were found to associate more with the emotionfocused coping approach than the problem-focused coping approach. This result is similar to that of a study in South Africa, in which the majority of coping mechanisms chosen by medical students consisted of emotionfocused styles such as positive reframing and religious coping [51]. A study from Germany also found emotionfocused functional coping strategies among their students, such as exercising and seeking support from friends and family [50]. The study by Thompson et al. also suggests that social support plays a major role in the coping mechanisms of medical students [3]. Emotional social support arising from human connection is instrumental in preserving positive coping mechanisms 
and students' wellbeing [54]. Moreover, the apparent improvement in coping mechanisms shown by fourth- and fifth-year medical students may result from these cohorts' increased social interaction as they enter clinical rotations.

Furthermore, a weak correlation was observed between academic performance (GPA) and resilience. We found that GPA was one personal characteristic that showed a very low correlation with resilience across student groups. Resilience can be seen as resulting from the successful interplay of personal and social factors in overcoming challenges, including challenges in medical study [52]. Academic performance may correlate with resilience through academic satisfaction and self-efficacy, which increase self-assuredness [11, 12]. This supports previous findings, which show statistically significant and positive correlations between academic self-efficacy and academic resilience [55]. Self-efficacy is also a significant predictor of academic performance (as measured by GPA) in higher education settings, although not necessarily in undergraduate medical education settings [56]. We did not assess students self-efficacy or academic satisfaction. We also could not assess the dynamics between students' resilience and their academic performance throughout the year, due to the limitations of our study design. Future studies should account for factors that might influence the correlation between resilience and academic performance, due to the multilayered and complex nature of resilience.

The regression analysis in this study indicates that $46.9 \%$ of resilience could be explained by coping mechanisms, personality, and academic achievement; these personal factors affect resilience variably across year levels (43.4-56.2\%). Our study finds that the year-4 students seemed to utilize their personal factors to foster resilience better than students at other year levels. Resilience itself stems from interaction between individuals (including their values, personal characteristics, and prior experiences) and external factors (including social support, learning environment, and cultural and ethical influences) [57, 58]. The dynamics of personal factors that predict resilience among students in our study seem to align with studies in other settings that have separately explored correlations between specific personal characteristics and resilience. Therefore, our study further illuminates the interplay between three personal factors (personality, coping mechanisms, and academic performance) for medical students' resilience. The adaptive coping mechanisms in our study showed the highest impact on medical students' resilience. An important aspect of adaptive coping is the use of instrumental and emotional support. Given that Indonesia has a highly collectivist culture, support from peers, family, and teams are generally always available [34]. One study reported that team support enhanced individual coping skills during stressful events and relieved emotional hardship; thus, it may also improve individual resilience [59].

Our study showed constant level of resilience across study years, which was affected by personal factors. However, it was known that resilience can be learned and improved overtime through a systematic intervention $[14,60]$. Therefore, the results emphasized the importance of facilitating students with periodic selfassessment of their coping mechanism, personality, academic achievement as well as significant experiences, while actively reflecting on those aspects to know themselves better. Such opportunities may help them to identify whether they are in needs for further support from peers or faculty members, hence increasing their resilience overtime. Our findings also strongly support efforts to provide personalized learning for medical students in the current era [61]. Identifying personal factors that impact resilience among medical students enables students to optimize their potential to overcome challenges in their studies. This also enables medical teachers and medical schools to provide necessary support for medical students, considering both personal factors and external challenges (e.g., curriculum, assessment, learning environment). It is equally important to further explore the external factors that affect resilience in this setting to further support medical students' resilience and wellbeing [62].

This study has several limitations. First, due to the limitations of a cross-sectional design, our results cannot explain the causal relationships between resilience and the personal factors being measured. Nevertheless, we have elaborated the dynamics between some personal factors and their correlations with resilience among medical students. Second, the personal factors we measured only partially predict medical students' resilience. Further research is needed to elucidate the relationship between medical students' resilience and personal factors such as self-efficacy or external factors such as social support and the learning environment.

\section{Conclusion}

The levels of resilience among undergraduate medical students were similar across years 1-6; these levels were comparable to resilience scores among undergraduate medical students in other settings, especially in Asia. Our results indicate that coping mechanisms, personality traits (according to the Big Five Personality Test), and academic performance may predict resilience. The adaptive coping mechanism seems to be highly important for cultivating resilience among medical students. This study strongly suggests the importance of identifying personal factors that support resilience in this population, as part of efforts to provide students with support and personalized learning. 


\section{Acknowledgements}

The authors would like to acknowledge all medical students who participated in this study. The authors would like to acknowledge Hervi Salsabila Mahkota Parentsia and Andhika Ridwan Achmadi who actively participated in the data collection.

\section{Authors' contributions}

Ardi Findyartini led the study, completed data analysis, and led the manuscript development. Nadia Greviana led the data collection, completed data analysis, and contributed to the manuscript development. Azis Muhammad Putera contributed in the data analysis and manuscript development. Reynardi Larope Sutanto contributed in the data analysis and manuscript development. Vernonia Yora Saki completed statistical analysis and contributed in the manuscript development. Estivana Felaza contributed in the data analysis and manuscript development. All authors approved the final version of the manuscript.

\section{Author's information}

Ardi Findyartini is an Associate Professor in Medical Education and is currently the Head of the Medical Education Department and the Medical Education Center - Indonesia Medical Education and Research Institute (IMER I), Faculty of Medicine Universitas Indonesia.

Nadia Greviana is an academic staff member and researcher at the Medical Education Department and the Medical Education Center - Indonesia Medical Education and Research Institute (IMERI), Faculty of Medicine Universitas Indonesia.

Azis Muhammad Putera is a research intern at the at the Medical Education Center - Indonesia Medical Education and Research Institute (IMERI), Faculty of Medicine Universitas Indonesia and is currently an undergraduate medical student at FMUI.

Reynardi Larope Sutanto is a research intern at the at the Medical Education Center - Indonesia Medical Education and Research Institute (IMERI), Faculty of Medicine Universitas Indonesia and is currently an undergraduate medical student at FMUI.

Vernonia Yora Saki is a research collaborator at the Medical Education Center - Indonesia Medical Education and Research Institute (IMERI), Faculty of Medicine Universitas Indonesia.

Estivana Felaza is a lecturer in Medical Education, an academic staff member and researcher at the Medical Education Department and the Medical Education Center - Indonesia Medical Education and Research Institute (IMER I), Faculty of Medicine Universitas Indonesia.

\section{Funding}

The study was funded by Universitas Indonesia (Grant number: NKB-1921/ UN2.R3.1/HKP.05.00/2019). The funding body has no role in the design of the study and collection, analysis, and interpretation of data and in writing the manuscript.

\section{Availability of data and materials}

The datasets generated and/or analysed during the current study are not publicly available due to conditions of participants' consents but are available from the corresponding author on reasonable request.

\section{Ethics approval and consent to participate}

All participants in this study provided informed consent prior to data collection. The participants were informed that their participation was completely voluntary and that the authors would ensure the confidentiality of the data provided in any of the reports that resulted from this study. The study protocol, was approved by the Research Ethics Committee of the Faculty of Medicine, Universitas Indonesia (No. KET-1299/UN2.F1/ETIK/ PPM.00.02/2019). All methods were carried out in accordance with relevant guidelines and regulations.

\section{Consent for publication}

Not applicable.

\section{Competing interests}

The authors report no competing interests.

\section{Author details}

${ }^{1}$ Medical Education Center, Indonesia Medical Education and Research Institute (IMERI), Faculty of Medicine Universitas Indonesia, Jakarta, Indonesia. ${ }^{2}$ Department of Medical Education, Faculty of Medicine Universitas Indonesia, Jakarta, Indonesia. ${ }^{3}$ Undergraduate Medical Program, Faculty of Medicine Universitas Indonesia, Jakarta, Indonesia.

Received: 12 October 2020 Accepted: 5 January 2021

Published online: 18 February 2021

\section{References}

1. Smith BW, Dalen J, Wiggins K, Tooley E, Christopher P, Bernard J. The brief resilience scale: assessing the ability to bounce back. Int J Behav Med. 2008; 15:194-200

2. Howe A, Smajdor A, Stöckl A. Towards an understanding of resilience and its relevance to medical training. Med Educ. 2012;46(4):349-56.

3. Thompson G, McBride RB, Hosford CC, Halaas G. Resilience among medical students: the role of coping style and social support. Teach Learn Med. 2016;28(2):174-82.

4. Osório C, Probert T, Jones E, Young AH, Robbins I. Adapting to stress: understanding the neurobiology of resilience. Behav Med. 2017;43(4):30722.

5. de Oliveira AC, Machado AP, Aranha RN. Identification of factors associated with resilience in medical students through a cross-sectional census. BMJ Open. 2017;7:e017189.

6. Banerjee Y, Akhras A, Khamis AH, Alsheikh-Ali A, Davis D. Investigating the relationship between resilience, stress-coping strategies, and learning approaches to predict academic performance in undergraduate medical students: protocol for a proof-of-concept study. JMIR Res Protoc [Internet] 2019;8(9) Available from: https://www.ncbi.nlm.nih.gov/pmc/articles/ PMC6754686/, [cited 2020 Sep 12].

7. Rotenstein LS, Ramos MA, Torre M, Segal JB, Peluso MJ, Guille C, et al. Prevalence of depression, depressive symptoms, and suicidal ideation among medical students: a systematic review and meta-analysis. JAMA. 2016;316(21):2214-36

8. Houpy JC, Lee WW, Woodruff JN, Pincavage AT. Medical student resilience and stressful clinical events during clinical training. Med Educ Online. 2017; 22:1320187

9. Ercan $\mathrm{H}$. The relationship between resilience and the big five personality traits in emerging adulthood. Eaurasian J Educ Res. 2017;70:83-103.

10. Foumani GHE, Salehi J, Babakhani M. The relationship between resilience and personality traits in women. J Educ Manag Stud. 2015;5(2):116-20.

11. Meneghel I, Martínez IM, Salanova M, de Witte H. Promoting academic satisfaction and performance: building academic resilience through coping strategies. Psychol Sch. 2019:1-16.

12. Burgis-Kasthala S, Elmitt N, Smyth L, Moore M. Predicting future performance in medical students. A longitudinal study examining effects of resilience on low and high performing students. Med Teach. 2019;41(10): 1184-91.

13. Eckleberry-Hunt J, Lick D, Hunt R. Is medical education ready for generation Z? J Grad Med Educ. 2018;10(4):378-81.

14. Farquhar J, Kamei R, Vidyarthi A. Strategies for enhancing medical student resilience: student and faculty member perspectives. Int J Med Educ. 2018; 12(9):1-6.

15. Hamou-Jennings FA, Dong C. Resilience training for healthcare providers: an Asian perspective. mHealth. 2016;2:25

16. Wu Y, Yu W, Wu X, Wan H, Wang Y, Lu G. Psychological resilience and positive coping styles among Chinese undergraduate students: a crosssectional study. BMC Psychol. 2020;8:79.

17. Peng L, Zhang J, Li M, Zhang Y, Zuo X, Miao Y, Xu Y. Negative life events and mental health of Chinese medical students: the effect of resilience, personality, and social support. Psychiatry Res. 2012;196(1):138-41.

18. Souri H. Hasanirad T (2011). Relationship between resilience, optimism and psychological wellbeing in students of medicine. Procedia-Social Behav Sci. 2011;30:1541-4.

19. Connor KM, Davidson JRT. Development of new resilience scale: ConnorDavidson resilience scale (CD-RISC). Depress Anxiety. 2003;18:71-82.

20. Carver CS. You want to measure coping but your protocol's too long: consider the brief COPE. IJBM. 1997;4(1):92-100.

21. Goldberg LR. The development of markers for the big-five factor structure. Psychol Assess. 1992;4:26-42. https://doi.org/10.1037/1040-3590.4.1.26. 
22. Ramdhani N. Adaptasi Bahasa dan Budaya Inventori Big Five. Jurnal Psikologi. 2012;39(2):189-207.

23. Blunch NJ. Introduction to structural equation modelling using SPSS and AMOS. Thousand Oaks, CA: Sage Publications Ltd.; 2008.

24. Cooper C, Katona C, Orrell M, Livingstone G. Coping strategies and anxiety in caregivers of people with Alzheimer's disease: the LASER-AD study. J Affect Disord. 2006;90(1):15-20.

25. Meyer B. Coping with severe mental illness: relations of the brief COPE with symptoms, functioning and well-being. J Psychopathol Behav Assess. 2001; 23(4):265-77.

26. Chen S, Chen T, Bonanno GA. Expressive flexibility: enhancement and suppression abilities differentially predict life satisfaction and psychopathology symptoms. Personal Individ Differ. 2018;126:78-84.

27. Lü W, Wang Z, You X. Physiological responses to repeated stress in individuals with high and low trait resilience. Biol Psychol. 2016;120:46-52.

28. Shi J, Chen Z, Yin F, Zhao J, Zhao X, Yao Y. Resilience as moderator of the relationship between left-behind experience and mental health of Chinese adolescents. Int J Social Psychiatry. 2016. https://doi.org/10.1177/002076401 6636910.

29. Allan JF, McKenna J, Dominey S. Degrees of resilience: profiling psychological resilience and prospective academic achievement in university inductees. Br J Guid Couns. 2014;42:9-25.

30. Brown DL, Tylka TL. Racial discrimination and resilience in African American young adults: examining racial socialization as a moderator. J Black Psychol. 2011;37:259-85.

31. Pidgeon AM, Keye M. Relationship between resilience, mindfulness, and psychological well-being in university students. Int J Liberal Arts Soc Sci. 2014;2.

32. Clauss-Ehlers CS. Sociocultural factors, resilience, and coping: support for a culturally sensitive measure of resilience. J Appl Dev Psychol. 2008;29:197212.

33. Clauss-Ehlers CS. Re-inventing resilience: a model of "culturally-focused resilient adaptation". In: Clauss-Ehlers CS, Weist MD, editors. Community planning to foster resilience in children York. NY: Kluwer Academic Publishers; 2004. p. 27-41.

34. Hofstede G. Culture's consequences: comparing values, behaviours, institutions, and organisations across nations. 2nd ed. Thousand Oaks, CA: Sage; 2010.

35. Reyes AT, Serafica R, Cross CL, Constantiono RE, Arenas RA. Resilience, acculturative stress, and family norms against disclosure of mental health probllems among foreign-born Filipino American women. Asian Pac Is| Nurs. 2018;3(3):80-92.

36. Hancock J, Mattick K. Tolerance of ambiguity and psychological well-being in medical training: a systematic review. Med Educ. 2020;54:125-37.

37. Bleakley A, Brice J, Bligh J. Thinking the post-colonial in medical education Med Educ. 2008;42(3):266-70.

38. Westerman M, Teunissen PW. Transitions in medical education. In: Walsh K editor. Oxford textbook of medical education: Oxford University Press; 2013.

39. Chen D, Lew R, Hershman W, Orlander J. A cross-sectional measurement of medical student empathy. J Gen Intern Med. 2007;22(10):1434-8.

40. Hojat M, Vergare MJ, Maxwell K, et al. The devil is in the third year: a longitudinal study of erosion of empathy in medical school. Acad Med. 2009;84(9):1182-91.

41. Ponnamperuma G, Yeo SP, Samarasekera DD. Is empathy change in medical school geo-socioculturally influenced? Med Educ. 2019;53(7):655-65.

42. Wahjudi JW, Findyartini A, Kaligis F. The relationship between empathy and stress: a cross-sectional study among undergraduate medical students. Korean J Med Educ. 2019;31(3):215-26.

43. Oshio A, Taku K, Hirano M, Saeed G. Resilience and big five personality traits: a meta-analysis. Pers Individ Differ. 2018;127:54-60.

44. Stajkovic AD, Bandura A, Locke EA, Lee D, Sergent K. Test of three conceptual models of influence of the big five personality traits and selfefficacy on academic performance: a meta-analytic path-analysis. Personal Individ Differ. 2018;120:238-45.

45. Judge TA, llies R. Relationship of personality to performance motivation: a meta-analytic review. J Appl Psychol. 2002;87:797-807.

46. Caprara GV, Alessandri G, Di Giunta L, Panerai L, Eisenberg N. The contribution of agreeableness and self-efficacy beliefs to prosociality. Eur J Personal. 2009;24:36-55.
47. Brown SD, Lent RW, Telander K, Tramayne S. Social cognitive career theory, conscientiousness, and work performance: a meta-analytic path analysis. J Vocat Behav. 2011;79:81-90.

48. Sanchez-Cardona I, Rodriguez-Montalban R, Acevedo-Soto E, Lugo KN, Torres-Oquendo F, Toro-Alfonso J. Self-efficacy and openness to experience as antecedent of study engagement: an exploratory analysis. Procedia Soc Behav Sci. 2012;46:2163-7.

49. Schmitt $N$. The interaction of neuroticism and gender and its impact on self- efficacy and performance. Hum Perform. 2008;21:46-61.

50. Erschens R, Loda T, Herrmann-Werner A, Keifenheim KE, Stuber F, Nikendei C, Zipfel S, Junne F. Behaviour-based functional and dysfunctional strategies of medical students to cope with burnout. Med Educ Online. 2018;23: 1535738.

51. van der Merwe $L$, Botha A, Joubert G. Resilience and coping strategies of undergraduate medical students at the University of the Free State. S Afr Psychiatry. 2020;26. https://doi.org/10.4102/sajpsychiatry.v26i0.1471.

52. Dunn LB, Iglewicz A, Moutier C. A conceptual model of medical student well-being: promoting resilience and preventing burnout. Acad Psychiatry. 2008:32:44-53.

53. Fergus S, Zimmerman MA. Adolescent resilience: a framework for understanding healthy development at the face of risk. Annu Rev Public Health. 2005:26:399-419.

54. MacArthur KR, Sikorski J. A qualitative analysis of the coping reservoir model of pre-clinical medical student well-being: human connection as making it 'worth it.'. BMC Med Educ. 2020;20. https://doi.org/10.1186/s12909-020-02 067-8.

55. Cassidy S. Resilience building in students: the role of academic self-efficacy. Front Psychol. 2015;6:1781.

56. Cassidy S. Exploring individual differences as determining factors in student academic achievement in higher education. Stud High Educ. 2012;37(7): 793-810.

57. Rutter M. Resilience as a dynamic concept. Dev Psychopathol. 2012;24(2): 335-44.

58. Tempski P, Martins MA, Paro HB. Teaching and learning resilience: a new agenda in medical education. Med Educ. 2012;46(4):345-6.

59. Zander M, Hutton A, King L. Coping and resilience factors in pediatric oncology nurses. J Pediatr Oncol Nurs. 2010;27(2):94-108.

60. Kreitzer MJ, Klatt M. Educational innovations to foster resilience in the health professions. Med Teach. 2017;39(2):153-9.

61. Quirk M, Chumley $H$. The adaptive medical curriculum: a model for continuous improvement. Med Teach. 2018;40(80):786-90.

62. Tempski P, Santos IS, Mayer FB, Enns SC, Perotta B, Paro HBMS, et al. Relationship among medical student resilience, educational environment and quality of life. PLoS One. 2015;10(6):e0131535.

\section{Publisher's Note}

Springer Nature remains neutral with regard to jurisdictional claims in published maps and institutional affiliations.

\section{Ready to submit your research? Choose BMC and benefit from:}

- fast, convenient online submission

- thorough peer review by experienced researchers in your field

- rapid publication on acceptance

- support for research data, including large and complex data types

- gold Open Access which fosters wider collaboration and increased citations

- maximum visibility for your research: over $100 \mathrm{M}$ website views per year

At BMC, research is always in progress.

Learn more biomedcentral.com/submissions 